\title{
Neurosurgical Research - Cutting Edge: Recent Advancements and Shortcomings in Neural Growth and Regeneration
}

\author{
Komal Sharma \\ Sir Run Run Shaw Hospital, Zhejiang University School of Medicine, Hangzhou, China \\ Email address: \\ k.sharma1604@yahoo.com
}

\section{To cite this article:}

Komal Sharma. Neurosurgical Research - Cutting Edge: Recent Advancements and Shortcomings in Neural Growth and Regeneration. Journal of Surgery. Vol. 4, No. 2, 2016, pp. 49-59. doi: 10.11648/j.js.20160402.20

Received: April 30, 2016; Accepted: May 19, 2016; Published: May 23, 2016

\begin{abstract}
Advancements in neurosurgical research have been central to understanding the underlying process of neural growth and regeneration (neurogenesis) and its application in the treatment and management of neurological pathologies. Recent work has shown the adult brain and central nervous system (CNS) to contain neuronal progenitors, precursor molecules as well as stem cells which play key roles in the new generation of neurones, astrocytes and oligodendrocytes. Whilst also challenging the previous dogma that no new neurones are born into the adult mammalian CNS and brain; this paper draws upon and presents an up-to-date picture of currently used "in vitro" and "in vivo" models for the study of novel techniques concerning the integration of neurones into existing neural circuitry. Breakthrough studies in Axonal Regeneration; The Application of Growth Factors; Stem cell grafting and proliferation; Synthetic materials technology; are just a few examples of key areas which have gained extensive interest, to ensure the successful clinical application of neurogenesis in various neurological pathologies. Challenges in the field are also addressed, where a future outlook to improve these novel techniques and potential areas of expandable research is also indicated.
\end{abstract}

Keywords: Biosynthetic Materials, Neural Growth, Neural Regeneration, Neurogenesis, Neurosurgery,

Neurosurgical Research, Surgery, Surgical Research

\section{Introduction}

Brain Trauma, psychiatric disorders and neurodegenerative diseases have posed enormous burdens not only in the neurosurgical department, but also on patient family members and in our society. Although prophylactic measures or palliative treatment for these disorders have been readily available; the aetiology and disease mechanisms behind neurological pathologies have been widely debated for the development of more effective treatment strategies [27]. There has been an increasing amount of evidence which indicates that adult neurogenesis (i.e. generating new Central Nervous System (CNS) neurones during adulthood) may be involved throughout the pathology and pathogenesis of different CNS disorders, and thus neurogenesis may be a potential target for treatment. Although, over the past decade, emphasis has been based on the clinical applications of new neurones as the "major player" in mediating treatment efficacy of neurological and psychotropic drugs in a cognitive setting; neurogenesis has also been debated over in terms of its reliability where it has been questioned if whether "An altered production of new neurones can under some circumstances cause the disorder itself?" [27]. Therefore the mentioned statement has come under some scrutiny over these ten years, as there has in fact been a substantial amount of published data regarding the basic in vivo and in vitro biology of neural stem cells (NSCs). The published material draws its main focus on records of the successful application of cell replacement therapy to improve a multitude of neurological pathologies, where the direction of treatment does not seem as bleak as it once was. Currently, there are several replacement therapies available for various human neurological, neurodegenerative disease and stroke. Furthermore, with respect to the application of neurosurgical research, discoveries made over the past 40 years, has resulted in an increased wealth of knowledge regarding stem 
cell biology for the use of neural growth and regeneration (neurogenesis) [29].

Adult neurogenesis is known as the generation of newly functional neurones from neural precursor cells, either resulting in the new addition or the full replacement of neurones of the CNS - either under physiological, pathological conditions, or both [27]. Neurogenesis in the brain of adult mammals has the ability to occur throughout life, where active adult neurogenesis is spatially restricted under normal conditions to two specific "neurogenic" regions in the brain, (i) the subgranular zone (SGZ) in the dentate gyrus of the hippocampus where new dentate granule cells are generated, and the (ii) subventricular zone (SVZ) of the lateral ventricles where new neurons are generated and then migrate through the rostral migratory stream (RMS) to the olfactory bulb $(\mathrm{OB})$ to become interneurons (Figure 1.) [10, $22,30,33]$. The capacity to replace lost neurones as a result of insult whether traumatic, surgical or due to impaired congenital development throughout the damaged regions of the brain, has been central in neurological and neurosurgical research in order to increase the successful outcome of both surgical and pharmacological intervention. With increased number of in vitro and in vivo experiments, the efficacious knowledge of how many neurones would be needed to fully replace a damaged region and mature into region-specific functional neurones to restore lost brain function has recently been more and more elaborated on. [32]. An example of such knowledge has heavy emphasis on "pre-cursor cell biology", where new life has been brought into the world of neural transplantation as well as the consideration of cellular replacement strategies to treat various diseases of the brain. This paper deals with and provides a critical analysis and an up-to-date picture of adult neurogenesis; including situations in which novel and prospective methods of neurogenesis have been successfully applied in pathological clinical contexts; and the future implications of neural growth and regeneration.

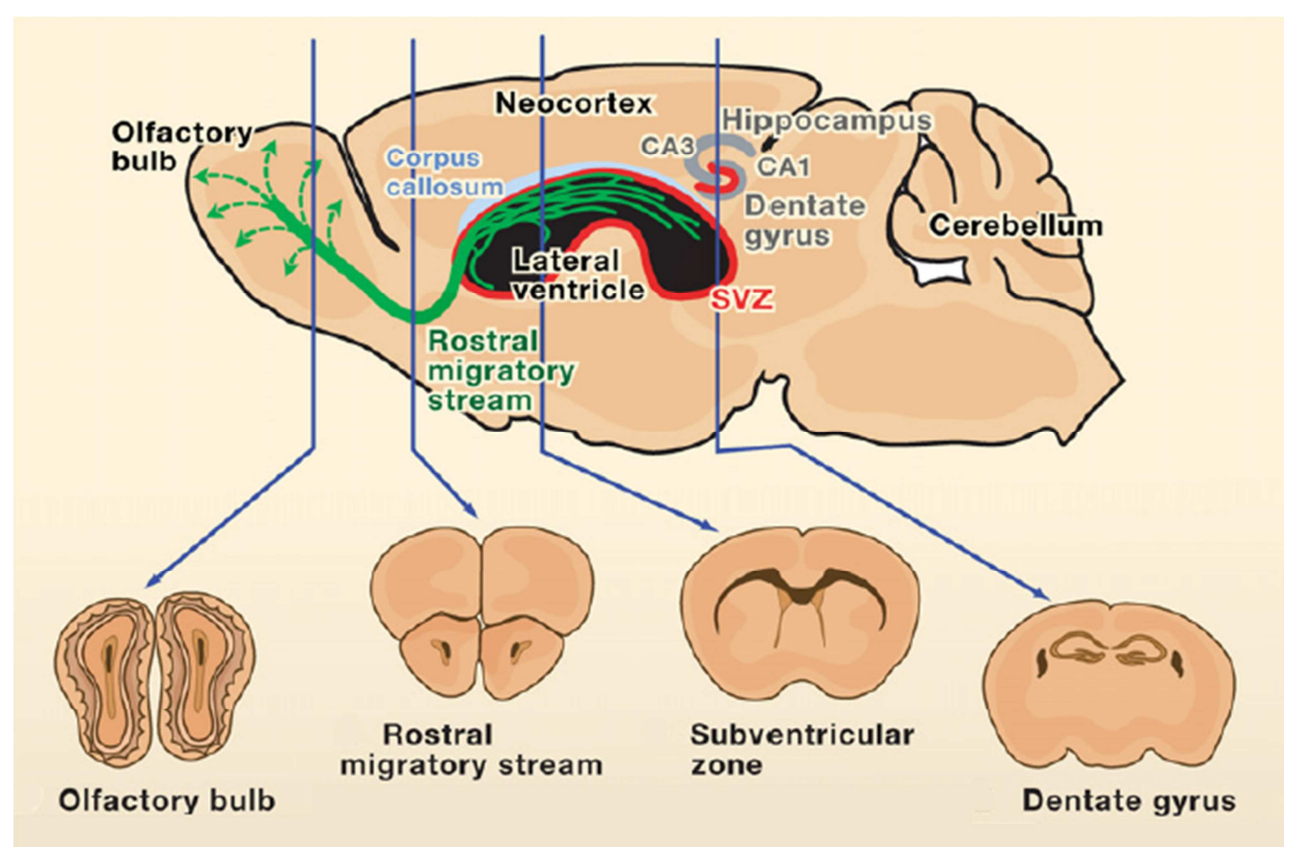

Figure 1. Neurogenesis in the Adult Rodent Brain - This figure depicts the sagittal and coronal views of the mouse brain in areas where neurogenesis occurs. Red areas indicate the germinal zones in the adult mammalian brain: the subgranular zone (SGZ) of the dentate gyrus in the hippocampus and the subventricular zone (SVZ) of the lateral ventricles. Neurons generated in the SVZ migrate through the rostral migratory stream and are incorporated into the olfactory bulb. Various significant structures in the rodent brain system has been emphasised in a study presented by Zhao et al, in 2008. Neurogenesis revealed by BrdU incorporation in the olfactory bulb, rostral migratory stream, SVZ, and dentate gyrus [33].

\section{Neurogenesis: In the Pathological \& Clinical Context}

Neurological diseases and brain injuries have been known to induce a progressive cascade of related events which in turn contribute towards events (not limited to): inflammatory responses, brain oedema, neuronal death, axonal injury radicalmediated damage, excitotoxicity, dysregulation of calcium homeostasis and mitochondrial dysfunction [27]. Excluding the two specific neurogenic regions of the brain (SGZ, SVZ), neurogenesis in other adult CNS regions of the body is generally believed to be very limited under normal physiological conditions, but it has also been confirmed that cell proliferation can be induced after injury [22]. Furthermore, much has been researched about properties and characteristics of neural precursor subtypes in the adult CNS, the supporting local environment and the sequential steps of adult neurogenesis, which may range from proliferative neural precursors to the synaptic integration derived from neuronal cells of the new-born [22]. Developments have also been rewarding as these areas of research have not only have provided significant answers to many fundamental questions about adult neurogenesis, but has also made a broad impact on 
general principles of stem cell regulation, neuronal development, structural plasticity, and disease mechanisms. These studies have also led to a number of controversies, intense debates, and conflicting conclusions and models that need to be independently expanded on in clinical research [22].

\subsection{Neurogenesis and Brain Injuries}

Reported studies in cases of brain injuries show the presence of a paradoxical positive feedback mechanism, by which the induction of neurogenesis is possible in post braintraumatic brain patients (i.e. ischemic stroke), where trauma results in immediate neurogenesis. This natural physiological process can be further enhanced through the aid of physiological growth factors. Research has also addressed the fate of neuroblasts after focal ischemia. For example, neuroblasts in the SVZ may migrate to injured areas such as the striatum [27]. This discovery in turn leads to the possibility of the SVZ being a steady reservoir for newborn neurones, in turn providing a potential therapeutic target.

Patients with traumatic brain injury (TBI) have different patterns of presentation where neuronal loss is focal and diffuse and often occurs as a result of necrotic and apoptotic mechanisms. Within these diffuse sites of injury, the hippocampus is known to be frequently damaged in humans, leading to defective learning and memory. However, once trauma has occurred, significant recovery also occurs where neurogenesis is thought of as one of the potential contributors towards recovery. This observation raises hope that TBI induced neurogenesis may serve as a means to not only replace damaged neurones but to also contribute towards the repair of neuronal circuits and overall neurological recovery [27].

\subsection{Neurogenesis and Neurodegenerative Disease}

The process of neurogenesis has been somewhat of a debate with respect to neurodegerative diseases. Different patterns of neurogenesis have been seen in patients with Alzheimer's disease (AD), Parkinson's disease (PD) and Huntington's Disease (HD). Patients with AD are known to occur with the most common cases of senile dementia. Neuropathological hallmarks of AD include neurofibrillary tangles (NFT) and senile plaques (being deposits of amyloid$\beta$ protein $(\mathrm{A} \beta))$. Patients with $\mathrm{AD}$ are seen to clinically present with loss of cognitive function and dementia which is progressive in nature. Motor functions are however, not disturbed in these patients. Within the early and progressive stages of $\mathrm{AD}$, due to the accumulation of plaques and tangles, the hippocampus is one of the first areas to be affected. Neurogenesis in these patients is seen differently than to what was observed in patients of brain injuries. The presence of $\mathrm{A} \beta$ accumulation in the hippocampus has been thought to be a links between $\mathrm{AD}$ and adult neurogenesis where a combination of both accumulating $A \beta$ and deteriorating neurones may significantly affect neurogenesis. An in vitro study demonstrated that $A \beta$ does indeed disrupt the differentiation of neuronal cells. Further in vivo studies in mouse models concluded that impaired hippocampus- dependent associative learning was correlated with reduced adult neurogenesis in the dentate gyrus [27]. Discrepancies in these results however have been faced with research that has shown the presence of enhanced neurogenesis by an AD-like pathology. Therefore, despite in-depth research, the functions of adult neurogenesis of patients in an AD-setting, remains a complicated conundrum of contradicted possibilities.

Individuals presenting with $\mathrm{PD}$ have a longstanding clinical history of progressive neurodegeneration through the loss of dopaminergic neurones in the substantia nigra (SN). Neuropathological factors include Lewy neuritis (LN) and Lewy bodies (LB) which contain these fibrillary and misfolded protein $\alpha$-synuclein - protein ( $\alpha$-syn deposits). Physiological neurogenesis in such patients has unfortunately failed to occur. Studies have showed negative results with respect to the production of new Dopamine (DA) neurones in the SN of mouse models with PD, even after treatment of growth factors (GF) [27]. This further suggests that newly generated dopaminergic neurones are extremely unlikely to be physiologically generated in the adult mammalian SN. A novel approach in this respect would be to drive research towards the production of a synthetic means of repairing the adult $\mathrm{SN}$ in patients with $\mathrm{PD}$.

Patients with HD (an autosomal recessive neurodegenerative disorder), are characterised through cytosine-adenine-guanine (CAG) expansion repeats within the Huntington protein encoding gene. Physiological effects on the brain would result in primary loss from gammaAminobutyric acid (GABA) medium spiny projection neurones in the caudate nucleus as well as deterioration of striatal interneurons. In clinical mouse models, it has been seen that a hostile Huntington-associated environment in the OB interferes with the survival and integration of new mature neurones in the process of neurogenesis. However, significant increases in cell proliferation in the subendymal layer (SEL) - adjacent to the caudate nucleus has also been reported, indicating the generation of neurones or glial cells in the SEL of the HD human brain [27]. These findings shed light on a strong indication of the positive correlation between natural physiological neurogenesis alongside progressive disease pathophysiology.

\subsection{Neurogenesis in Psychiatric Illness}

Clinical depression has been described as the first psychiatric disorder as "pro-neurogenic" as well as in patients with schizophrenia. In clinical depression, mechanisms underlying the physiological response to upregulate neurogenesis can be associated with stress and pharmacological intervention. Several studies have specified a strong connection between negative regulation of neurogenesis and stress. The stress inducing hormone corticosterone is seen as the main barrier between reducing levels of stress and the promotion of neurogenesis. Therefore administration of psychiatric medication for clinical depression (e.g. use of anti-depressants) in conjunction with stress-reduction activities (e.g. exercise) was seen to reduce stress levels and in turn elevate pro-neurogenic capability 
[27]. Studies including patients with anxiety and mood disturbances also showed positive results of pro-neurogenic activity, upon administration of Selective Serotonin Reuptake Inhibitors (SSRI's). Study groups consisting schizophrenic patients have also been recorded show a positive response towards pharmacological intervention where atypical antipsychotics such as clozapine and olanzapine increased the proliferation of new cells in different brain structures (such as the pre-frontal cortex, dorsal striatum, and hippocampus). Haloperidol, a prototypic antipsychotic was also found to increase neurogenesis in schizophrenic animal models [27].

\subsection{Peripheral Nerve Injury}

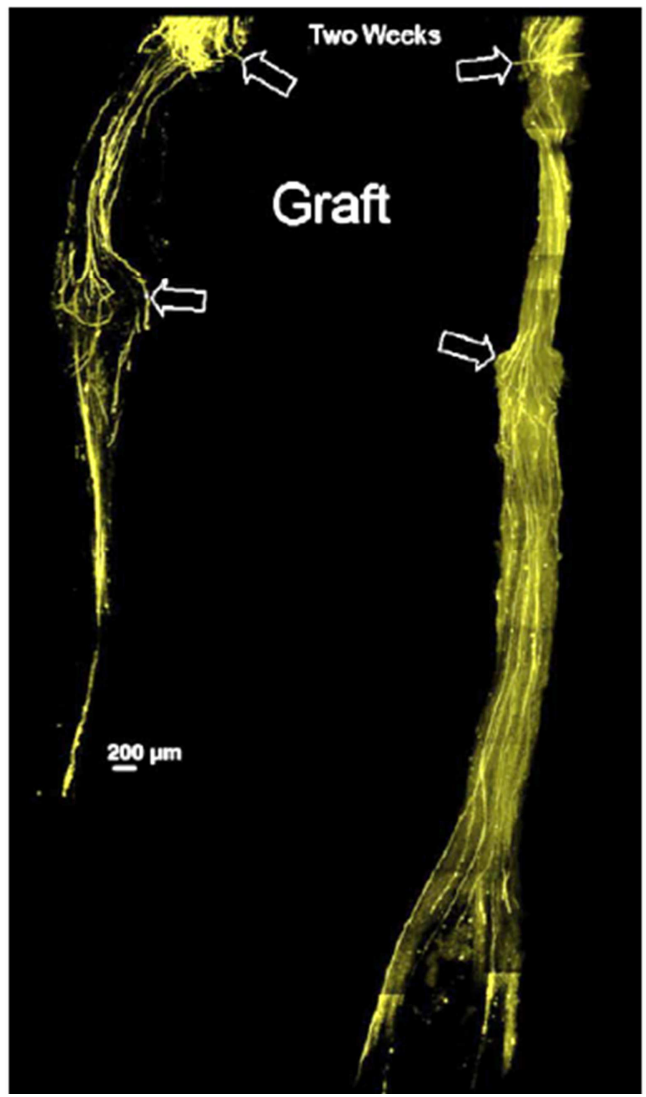

Figure 2. Peripheral Axonal Repair - Low magnification images of two common fibular nerves are displayed to show the effect of post-surgical rehabilitation through continuous training on the regeneration of cut/severed axon (right), when compared to axons with no post-surgical rehabilitation (left). Each nerve had been cut and surgically repaired 2 weeks earlier using a ca. $3 \mathrm{~mm}$ graft from a wild type donor mouse. The boundaries of each graft are indicated by arrows. The overall image of each nerve is a montage constructed from the paper presented by Deumens, et al. in 2010 from images of several microscope fields, each taken at the same depth through whole mounts of the nerve. It can be seen by 2 weeks after nerve repair, any residual fluorescence in the original distal stump (distal to the graft) due to the degeneration of the host axons has disappeared. Fluorescence found in this region thus represents profiles of regenerating axons that have grown entirely through the grafts. The nerve on the right came from a mouse that had received treadmill training after the nerve repair. The nerve on the left was from a mouse that was unexercised [7].

Patients with peripheral nerve injury (PNI) include approximately $3 \%$ of trauma patients [7]. Not only does PNI debilitate patients, but also compromise their quality of life, where the functional outcome of PNI depends on the severity of the injury from neurapraxia and axonotemesis (e.g. nerve crush). Patients presenting with nerve crush are shown to have better prognostic outcomes in comparison to neurapraxia, where a loss of motor function would be observed. Preservation of axonal activity however, would be observed in patients with neurapraxia including sensory response to heat, cold, irradiation or electrical injuries. These responses may majorly be attributed to mechanical stress as a result of concussions, compression or traction injuries and is further associated with a relative degree of ischemia. Additionally, with severe instances of neurapraxia are seen with local myelin abnormalities or even segmental demyelination. Symptoms as a result of axonal interruption may be manifested as nerve pinching, crushing or prolonged pressure. As neurogenesis is rare if not non-existent in peripheral of nerve injuries; minimal loss of both sensory and motor neurones can only be achieved through immediate and early surgical repair of the damaged nerve [7]. Treatment is solely dependent on surgery where recovery can be achieved given the right procedure and post-surgical rehabilitation (Figure 2). Various methods that are currently available for ensuring successful outcomes of neurogenesis shall be elaborated on in the next section of this paper.

\section{Novel Cellular Modifications in Neurogenesis: Clinical Trials and Tribulations}

\subsection{Axon Regeneration}

Where impairment of motor neurones is concerned, especially in the case of PNI, restoring neurological function to a damaged peripheral nerve separated by a gap requires axonal regeneration. Importantly, there are three variables of peripheral nerve traumas which includes (i) the length of the nerve gap; (ii) the time between nerve trauma and repair (iii) the age of the patient/subject [16]. Recent literature has suggested that sensory nerve grafts are the "clinical gold standard" for bridging nerve gaps promotes reliable axon regeneration. It must be noted that although the use of the autologous nerve graft is regularly mentioned in the literature as "the gold standard", in practice, such nerve grafts alone often support a far from perfect functional outcome. More than one novel nerve repair technique must therefore encompass utilization for effective treatment measures and an overall successful clinical outcome.

Currently there are three novel nerve repair techniques which have been clinically tested and that are able to induce axonal regeneration and neuronal recovery clinically under conditions where other techniques may have proven ineffective: (i) The first are conduits containing a sensory nerve graft with pure fibrin: This technique has been applied clinically in a patient where damage to the radial nerve was observed. The applied technique presented with $100 \%$ 
sensory and motor function in the patient, in which a sensory nerve graft surrounded by a collagen tube filled with pure fibrin, was inserted within the radial nerve gap. This surgical measure, when applied was showed to help enhance axonal regeneration. The mechanism behind the regeneration of these axons was made possible due to neutrophic factors released by graft Schwann cells by transforming the fibrin matrix into a potent promotor of axon regeneration (Reyes, O. et al, 2007). The resultant radial injury was fully recovered and no functional differences were apparent between the two hands; (ii) The second novel repair technique surrounds conduits containing a sensory nerve graft plus autologous platelet-rich plasma: Studies were conducted where a sensory nerve graft was surrounded by a collagen tube filled with autologous PRP (Platelet Rich Plasma). Again its clinical application in nerve repair yielded highly positive results. Of the 15 repaired nerves, $100 \%$ of the patients recovered completely - from limited nerve function to complete sensory function, and $93 \%$ developed limited motor function - to complete motor function. This technique is shown to induce axons to regenerate significantly longer distances and under conditions where sensory nerve grafts are not effective [16]; (iii) finally, the third novel repair technique includes conduits containing only autologous platelet-rich plasma. Clinical studies involving the application surrounding this technique had been based on a theory where it was hypothesised that bridging nerve gaps with a collagen tube filled with only autologous PRP would induce extensive axon regeneration and recovery; that too even in the absence of a sensory graft. When clinically applied, of 9 repaired nerves, $100 \%$ of the nerves developed limited to full sensory recovery, while $89 \%$ developed limited to full motor recovery [16]. These results are therefore highly significant in these outcomes to show that PRP is not only more effective than sensory nerve grafts, but can also induce axon regeneration and full neurological recovery under conditions where axons would normally not regenerate - as seen in chronically de-nervated distal nerves and in older patients for example. Based on these results, with respect to the patient's age, the remarkable efficacy of nerve grafting is further exemplified by a 58-year- old subject who recovered from limited neurological function although the nerve gap was $12 \mathrm{~cm}$ in length and the nerve was repaired 3.25 years post-trauma [16]. Additionally, the use of luminal matrix fillers has been shown as promising where the incorporation of either GFs or accessory cells which aid in the regenerative process can be loaded into a luminal matrix [24]. These matrices will function to provide structural support within the microenvironment, an act as a scaffold to help guide regenerating neurones in the process of growth and repair.

Further studies are now required to determine which of these methods can induce the most reliable outcome where both $100 \%$ axon regeneration and neurological recovery can be expected. Furthermore, it is important to know whether these techniques can be made effective when used in more devastating and debilitating neurological dysfunctions where sensory neurons as well as axons would require generation at the level of the central nervous system as well as in neurogenic regions of the brain.

\subsection{The Application of Growth Factors}

Research surrounding the effects of Growth Factor (GF) treatment has also demonstrated to be highly efficient for the regeneration of adult striatal neurones, following ischemia with its long-term effectiveness and concomitant recovery of motor performance (Figure 3). This novel approach, given the success of its application, yet still has its shortcomings as a better understanding of underlying molecular processes are required in order to contribute to the development of efficient regenerative therapy in patients with brain injuries [32]. Clinical trials have been conducted where the use of growth factors prove to be more effective when used in conjunction with other cell-based therapies, such as stem cells, than just on its own.

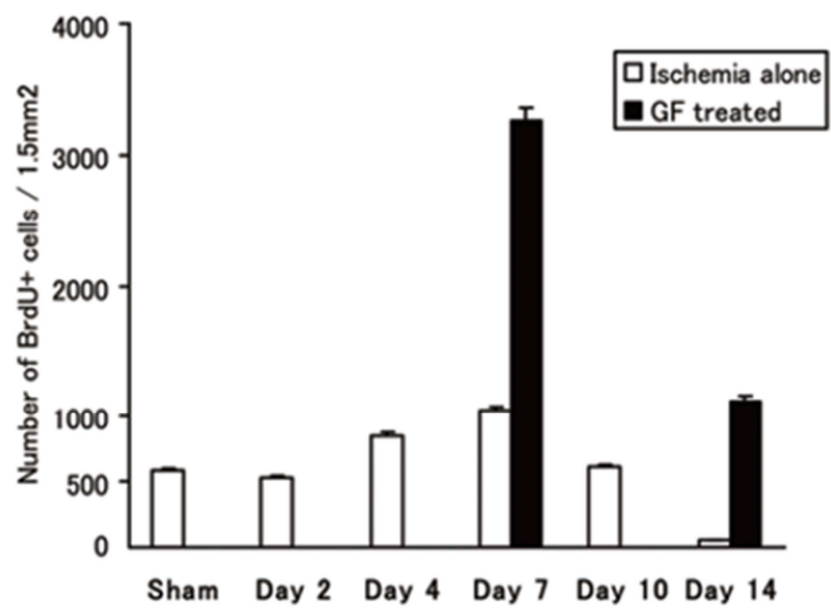

Figure 3. Ischemic injury increases cell proliferation in the SVZ - Bar graph shows the number of Bromodeoxyuridine (BrdU)-positive cells after ischemia alone or following GF treatment (4 rats in each group). In the study conducted by Yoshikawa et al, in 2010; the BrdU was intraperitoneally injected for 2 days before the rats were killed. It can be interpreted from this graph that ischemia-induced cell proliferation peaked on Day 7, which was further increased by GF treatment [32].

\subsection{Stem Cell Grafting and Proliferation}

Grafting of neural stem cells in the mammalian central nervous system has been performed for over a decade or two from research to bedside treatment, especially in the application of neurological disorders such a Parkinson's disease, Huntington's disease, stroke and spinal cord injuries [29]. Pathologically, stem cell therapy and regenerative approaches can be divided into various categories which heavily depend upon the main molecular targets of interest. These diseases include those which occur as a result of acute injury, chronic inflammatory and immunologically mediated conditions, chronic neurodegenerative disorders, and congenital neurological diseases. For these purposes, many other types of cells are also being used for their application in the treatment of neurological diseases and stroke [2, 21, 29]. In terms of stroke, a few studies have also evaluated the 
possible beneficial effect of the administration of stem cells in the early stages of stroke. It was seen that intravenous administration of allogenic mesenchymal stem cells (MSCs) from adipose tissue in patients with acute stroke could be clinically applied as a safe therapy for promoting neurovascular unit repair and consequently supporting better functional recovery. This study was performed on a patient who happened to be present in the first reported phase II clinical trials by Diez-Tejedor, E. et al in 2014 [8]. It was suggested that allogenic MSCs could be viewed as an alternative therapy as it has been demonstrated in their study that allogenic MSCs lack HLA-class II antigens, where no subsequent allergic reactions would be experienced; other than a regular classical inflammatory response or complete graft rejection. In addition, this therapy has also not been associated with the development of tumours where the benefits of using MSC in neurogenesis further outweigh its limitations.

NSCs can be sourced from skin, embryonically, bone marrow and adipose-derived mesenchymal, induced pluripotent stem cells (iPSCs), foetal and directly from the adult nervous system. All these sources have proved to be potent for the treatment of several neurological pathologies. For example, MSCs derived from different sources in the body have been successfully used for neurogenesis of dopamine neurones in the treatment of PD [29]. With respect to their source, Stem cells also exhibit two defining characteristics, the capacity of self-renewal through cellular division and the capacity for generating specialised cell type(s) through differentiation. Cells which have been shown to be capable of long-term expansion and differentiation into neurones and glia have been derived from adult rodent brains [22]. Limitation of such models exist as the extraction process is very time consuming where culture length can be long, leading to a risk of cellular reprogramming and expand the capacity of endogenous cells. However, as previously mentioned, positive results can be obtained where lineagerestricted neural progenitors can be cultured after exposure to Growth Factors (GF). This can result in the potential acquisition of neuronal regenerative properties which may had not been previously seen in vivo. For example, in traumatic brain injury patients, it has very recently been seen that neural progenitor cell transplantation shows highly positive neuroprotection abilities, further enhancing hippocampal neurogenesis and cognitive outcome [3]. Their highly successful application is mainly attributed to their intrinsic advantages, including the secretion of neurotrophins (which are critical for neuronal survival and repair). These stem cells grafts yielded greater positive in vitro results regarding neuronal survival if NPC's were to be genetically modified to secrete synthetic, human multineutrophin (MNTS1), Growth Factor (GF) - sub cloned into a lentivirus vector [3].

However, as successful as these methods are proving to be, drawbacks in these methodologies once again do exist. There is still need of getting more suitable cell sources for in vitro and in vivo trans-differentiation to produce accurate phenotypes. In clinical application of stem cells, importance should be drawn upon screening NSCs for bacterial, viral, or fungal contaminants, in order to avoid life threatening complications which may arise from neurosurgical grafting procedures. Furthermore, complete media replacement prior to grafting, with saline or $\mathrm{Ca} / \mathrm{Mg}$ free phosphate buffered saline (PBS), in order to sustain cellular viability and integrity [29]. With this caution in view, there are examples where treatment through stem cell grafting has not always proved be successful in a clinical setting. One such example indicates that stem cell grafting of PNI's has yielded significantly poor results as the optimal environment for axonal regeneration relies on the synthesis and release of many biochemical mediators that are temporally and spatially regulated with a high level of incompletely understood complexity. In this respect Schwann Cells (SCs) have emerged as a key player in this process. Uses of stem cells have clinically shown the ability to increase the number of SCs and prolong their ability to support surrounding neuronal structures and promote regeneration. Additionally, implanting grafts of stem cells with the ability to differentiate into SCs may have the ability to rescue and replenish populations of chromatolytic and apoptotic neurons following axotomy; and to help preserve injured tissues such as denerved muscles (Figure 4) [12]. The most superior outcome of SC differentiation has been observed by placing scaffolding stem cells in a culture medium which is then delivered through the epineurium with aid of natural conduits (three main conduits as previously discussed in axon regeneration). Drawback and limitations may only be present in the context of genetic manipulation, cell instability and increased risk of tumorigenesis [12].

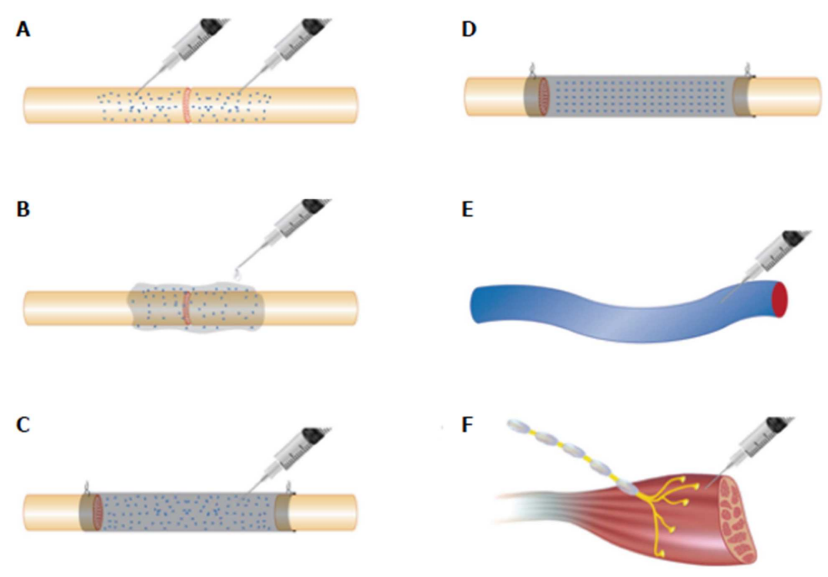

Figure 4. Methods of stem cell delivery - A. Stem cells are directly injected into nerve endings or bridging nerve grafts; B. Stem cells are suspended in matrix and are injected around the nerve rather than into the substance of the nerve; $C$. Nerve gap model using a conduit as the bridging method. Conduit lumen can be injected and filled with cells suspended in scaffold; $D$. Nerve gap model using conduit as bridging method. Conduit has been cultured and seeded with stem cells. Lumen of conduit may also be filled with cells. Hollow conduits may have internal framework added. This framework may also be seeded with cells; E. Systemic injection of cells. Stem cells have been shown to home to the site of injury; F. Cells may also be injected into neuromuscular junctions of peripheral denerved muscles [12]. 
As well as peripheral nerve neurogenesis, extensive research has been conducted to apply feasibility of stem cell grafting in neural tissues of the brain. NSC niches were observed to be located along the entire extent of the ventricular system, including in the midline, CVOs and along the $3^{\text {rd }}$ and $4^{\text {th }}$ ventricles [20]. These cells are seen to be capable of widespread neurogenesis and gliogenesis, in particular after exposure to injury or other various inductive stimuli. A key example of such stimuli are GF's which have been shown to travel to these niche stem cell areas throughout the brain via the CSF and through the BBB [19]. Thus, the infusion of a GF directly into the lateral ventricle in turn stimulates stem cell proliferation and differentiation at multiple stem cells niches along the ventricular system. Together, these activities may contribute towards the "spontaneous" functional recovery in patients recovering from stroke. Other novel techniques such as the paracrine hypothesis have also inspired an alternative outlook on the use of stem cells to regenerate neural tissue [19]. It has been seen that significant repair of the injured brain may be achieved by directly injecting the substance secreted by stem cells (secretome) to the lesion, rather than implanting stem cells themselves for direct cell replacement. The stem cell secretome (SCS) includes a combination of components such as cytokines, chemokines and growth factors, mitogens to promote neurogenesis; and has therefore gained increasing attention in recent years because of its simple application and multiple implications for the repair, restoration or regeneration of injured neuronal tissues [9]. Likewise, application of a combination of different factors has been proposed to not only be sufficient to increase the number of progenitor stem cells, but it may also increase the level of long term cell survival [5].

In demyelinating diseases such as multiple sclerosis (MS), however, discrepancies exist as previous studies have described that spontaneous re-myelination of the hippocampus was largely absent. Notably, it has been shown that differentiation of NSPCs into oligodendrocytes can be achieved within the adult hippocampus through retroviral overexpression of proteins (Ascl1, Olig2, or Sox10 TFs), which are known to play pivotal roles during brain development to induce oligodendrocyte lineage specification and maturation [4]. Additionally, the proven use of endogenous NSPCs for glial repair within the hippocampus has also been supported. However, this technique still remains in its analytical phases and is still unsuitable to apply clinically. Current trials however, have now been underway in the field where neural stem cells have been assessed against biophysical and biochemical parameters to ensure the successful growth and development of NCS. This turn, would then prove the effective recovery of patients as the biophysical parameters contain the mechanical properties and architecture of the ECM and human ground substance - which together form the fundamental foundation for tissue re-modelling. The biochemical parameters are composed of the chemical and bioactive cues originating from the soluble cytokines and growth factors released by the adjacent cells, cell adhesion molecules, and extracellular matrix molecules [31].

Limitations of applying these methods clinically still remains a challenge in other respects, as precise control of NSCs self-renewal and lineage commitment, structural remodelling of differentiated NSCs, neural reconnection, and correct transmission of nerve signals, are still major obstacles to achieving functional recovery. Use of advanced biomaterials is therefore an emerging area of further research in neurosurgery, which shall be discussed next. These synthetically produced constituents can help provide a more biomimetic micro- environment and significantly contribute to nerve repair and regeneration [31].

\subsection{Biomaterial (Synthetic) Technology}

The use of biomaterials have been shown to provide a functionalised scaffold for CNS tissue engineering and regeneration which helps bio-mimic NSCs niches, and in turn, regulates their fate of differentiation. One promising synthetic material which has the potential to be used as a conduit with differentiation of NSCs is the Hyaluronic Acid (HA) Hydrogel System [18]. Just as promising as it has been for over a decade, this technique of tissue engineering has been expected to function as a good tool for lesion repairs, not only in the brain but also successfully in other kinds of tissues and organs, such as skin, muscle, tendon and blood vessels [14]. HA being a widely distributed natural molecule in tissues, plays a large role in promoting cell motility and proliferation by integrating with receptors on the cell surface (Figure 5). Once made to be hydrogels and implanted into defects of the cerebrum, they function to assist induction of CNS regeneration in trauma patients. When observed at an ultrastructural level through a scanning electron microscope (SEM), HA hydrogels successfully act as scaffolds in the presence of laminin to support angiogenesis, permit cell migration to reach areas of lesions and defects to further prevent the formation of glial scars during neurogenesis [14]. More recent progress since, has been addressed by $\mathrm{Li}, \mathrm{X}$. et al in 2012, where use of transplanted NSCs within engineered hydrogels is a promising treatment for CNS injuries and disease [18]. Not only would these Hydrogels be used as a support scaffold, they can also provide a means of a precise delivery system of cell regulatory signals, thus inducing stem-cell fate in vivo. Their high plasticity has also been seen as an attractive feature for integrating into neurosurgical procedures. The ability of certain in situ hydrogels to crosslink, not only minimizes the invasiveness of the surgery, but allows for the formation of any desired shape at the injury site as well as strengthens the tissue-hydrogel interface [1]. The challenges faced to be overcome however, will be to integrate the building blocks of these complex systems, including the physical properties of hydrogels, the spatial and temporal control of signalling molecules, and the presentation of key ECM motifs, into modular and scalable platforms for clinical translation [18]. 

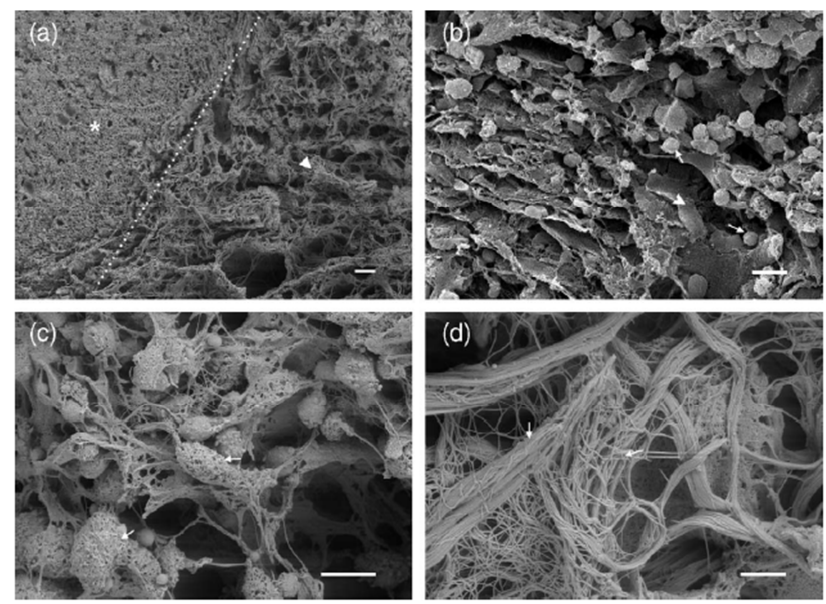

Figure 5. Scanning Electron Microscopy (SEM) examination of post 6-week surgical implantation sites - Research by Hou, S. et al in 2005, shows the structure of hydrogels filled in the defects of the cortex on a coronal section. (a) box in the upper left shows a low magnification assay revealing hydrogels (on the right of dotted line, arrowhead), that have merged with the normal tissue (on the left of dotted line, asterisk) of the brain and reestablished the anatomical continuity of the tissue. (b) In the internal structure of the porous hydrogels, the three-dimensional network (arrowhead) seen to be invaded by ample cells (arrows) in the group of modified and finally (c) are unmodified hydrogels. Bundles of like collagen (arrows) can be seen between invaded cells in the grafts under high power (d). Scale bar $=40 m(a) ; 10 m$ (b and c); $2 m$ (d) [14].

In the case of peripheral nerve repair, developments in tissue engineered nerve grafts have shown promise for the use as a part of the neurosurgical setting. Polymer based neural scaffolds encompass non absorbable neural scaffolds and bio-degradable/bio-absorbable neural scaffolds. Non absorbable scaffolds typically include silicone which has been clinically used in microsurgical methods for median and ulnar nerve injuries. Successful application has yielded positive results where a 5-year follow up showed no long term adverse outcome, except less severe cold intolerance. Better results have been seen in ulnar nerves than in median nerves. Another type of non-absorbable neural scaffold is the expanded polytetrafluoroethylene (ePTFE)-based Nerve Guided Conduit (NGC) [13]. When applied for the repair of a 29-mm ulnar nerve gap in a female patient; once again resulted in good recovery of both motor and sensory functions. With respect to Biodegradable and bio-absorbable neural scaffolds, these are made up from synthetic polymers. So far, the largest body of clinical data is concerned with application of Neurotube ${ }^{\mathrm{TM}}$ poly (glycolic acid) in facial neve repair yielded an overall of $14.2 \%$ excellent functional recovery, $57.2 \%$ good and $28.6 \%$ poor, respectively [13]. The NeuraGen $^{\mathrm{TM}}$ collagen NGC made up of type I collagen is also related to abundant clinical data. A retrospective clinical study reporting the tubular repair of the brachial plexus birth injury yielded results of excellent patient recovery 2 years post-surgery [13]. Similar results have been seen with repair of lingual and inferior alveolar nerve injuries. These results prove the extent of potential seen with this form of technology. If underlying molecular mechanisms are further explored and studied, polymer based neural scaffolds can be used where a drug delivery system is concerned as they can function as a strategy for the controlled release of growth factors, if cellular therapy is considered for use alongside.

Emerging nanotechnology approaches have also been considered for tissue engineering in peripheral nerve regeneration. This has been achieved through the use of scaffolds with functionalised cell binding domains, as well as use of guidance channels with cell-scale internally oriented fibres with potential of sustained release of neurotropic factors. Natural materials such as collagen, gelatine laminin and chitostan have been used in clinical models as electrospun scaffolds for nerve reconstruction [6]. However, due to their natural composition; their weak mechanical properties can limit their application as nerve guide conduits [15]. Therefore, attention is placed on use of collagen based matrix for a more successful outcome. Use of these matrices (Natural, synthetic and semi-synthetic) has been successfully applied not only in peripheral nerve regeneration, but also in spinal cord healing and scar formation $[6,28]$. However, their functions are more inclined to remodelling than promoting neurogenesis.

Although benefits outweigh the limitations of biomaterial usage in a neurosurgical setting, limitations to these methods still exist. Careful choice of biomaterials must be used if choice of a synthetic grafting technique is employed. A patient's immune status and immune system responses must be thoroughly assessed as studies have shown underresearched biomaterials to invoked high activation of the primary human dendritic cells, suggesting some materials would be unsuitable for safe routine in vivo implantation [23]. This further highlights the importance of testing, as without stringent testing in vitro, great time and expense would be spent taking novel materials forward when clearly they are not suitable for these in their current incarnation.

Finally, an important point must be considered in terms of accuracy of graft placement and delivery of cells and cellular factors. Further elucidation of the path-finding mechanisms of axons is necessary for developing more efficient methods that will enable nerve regeneration to better mimic natural process of neurogenesis [13]. Overall, nerve regeneration is highly complex whether in the brain, CNS or PNS, which requires tissue engineered nerve grafts to equally provide a close similarity to native nerve tissues, both in structure and in function. This area of research is highly sophisticated which would need great understanding on underlying molecular mechanisms to replace nerve tissue completely rather than regenerate it by natural means. Hence these procedures show great potential to be expanded on in the future, prior to clinical application.

\subsection{Other Breakthrough Techniques}

Hippocampal neurogenesis for the treatment of mood disturbances and anxiety has been yet another "sought out" field of research in the developments of targeted treatment and replacement of neurones in neurodegenerative diseases. Regnell, C.E., et al., 2012, mentioned in their review that one of the major determinants of adult hippocampal neurogenesis is hippocampal-dependent learning [25]. It was shown that 
receptor activation had increased adult hippocampal progenitor cell proliferation and differentiation. This activation was observed as a result of the adult brain being influenced by experience and synaptic activity. However, the major challenge in these circumstances has been to demonstrate the causal relationship between disrupted adult neurogenesis and deterioration of cognitive ability. Earlier approaches were made to address such shortcomings through the use of antimitotic drug treatments and irradiation, which effectively blocked proliferation of progenitor cells during neurogenesis but had substantial side effects, such as general health deterioration and inflammation, which complicated the interpretation of behavioural studies. Recent studies using transgenic mouse models involved the expression of suicide genes driven by various neural stem progenitor cells (NSPC)specific promoters. This "Neil3"/mouse model, would typically display defective DNA damage repair, diminished NSPC proliferation, and aberrant behaviour [25]. Such model creates an excellent basis for further studies of the casual relationships formed amongst cognitive performance, oxidative damage, and neurogenesis.

\section{Future Outlook}

Looking at the progress in research which has been presented over the recent decade, it can be seen that substantial advances have been directed towards developing surgical techniques in cell and tissue transplantation/modifications, material science including matrix design, surface modification, genetic and molecular therapy, as well as managing the rehabilitation of the patient.

Furthermore, emphasis of clinical research and application has been placed on stem cell differentiation. For example, it has been reported that mesenchymal stem cells (MSCs) can be differentiated to glial-type cells for PNI's in vitro via induction with various mitogens [7]. Given the apparent plasticity of such stem cells and their presence in adult tissues, it is likely that these cells will play roles in future neural tissue engineering, especially in the neurosurgical setting. Furthermore, embryonic stem cells and pluripotent cells will also have a strong impact on advances toward finding a suitable alternative to autologous nerve grafts. However, shortcomings as discussed can also be experienced when considering their application in conjunction with other components. For example, it will be difficult to define the ideal dosing of growth factor(s) (if used) as well as the timing of growth factor release on stem cells [7]. Future developments of implantable devices which combine neuronal modification with recognition domains for growth factor attachment should therefore be considered on being expanded in research. Although it is widely assumed according to medical literature that such strategies will improve nerve regeneration, it is important to consider the possibility that strategies may also ignore the cell body response of axotomised populations of neurons, which might not result in the optimal degree of response nor recovery.

It appears that neuronal precursor cells, although present in few numbers, might be much more widely distributed in the parenchyma of the adult CNS than previously thought. Another relatively unexplored theory in research is the possibility of cellular re-population within the mammalian CNS where the de-differentiation of seemingly mature cells and subsequent re-population of damaged area. [10]. Furthermore, wide distribution of pre-cursor cells within the mammalian CNS might include those that exist as differentiated cells, expressing mature neural markers and retaining the ability to differentiate into neuronal subtypes. This would be possible given the right conditions and orchestrated, regulated set of molecular signaling, through the "Wnt", "Tlx", "Disc1", "FMRP" and "Hypoxia Inducible Factor-1" (HIF-1) signalling pathways which are important for adult neurogenesis. [10, 11].

In addition to the potential of pre-cursor cell proliferation, less research has also been published on the functional significance of olfactory neurogenesis, even if consistent changes in olfactory behaviour has been reported, following the reduction of neurogenesis [33]. The ability to decipher the functional role of neurogenesis will not only provide clinicians with the necessary knowledge with regard to both olfactory and hippocampal brain circuit systems, but will more importantly allow us to focus on development of novel treatments to successfully manage and conquer the degree of devastation as a result of neurological insult in humans.

The use of biomaterials, as discussed, has transformed into a major area in research with space for extensive future development. There is no doubt that each single biophysical or biochemical property of biomaterials will have direct regulatory effect on NSCs fate and therefore should be considered when designing the applied scaffolds. From the viewpoint of biomaterials, future endeavours in injured brain regeneration should focus on how to optimize the combination of base materials and regulatory cues and exert the synergistic effects of different types of biomaterial parameters in a controllable manner [31].

In terms of prognostic value and clinical work-up, future rehabilitation of patients should not be ignored, especially if recovering from nerve trauma and injuries to the brain and spinal cord. It is known that in nerve injuries, such a PNI, the rehabilitation of patients may add a significant contribution to a faster and more efficient recovery [7]. Furthermore, with respect to treatment measures, timing is heavily attributed to successful outcomes. For example, a major reason for the failure of neuroprotective treatment in an acute injury occurs when there has been significant need to start immediate treatment. In an acute clinical setting, cell-based therapeutic approaches have been seen to provide a better option. Additionally, NSCs from various sources have shown effectiveness in improving motor function in several neurodegenerative diseases and stroke in animal experiments and clinical trial studies [29]. However, short-comings are also present in the application of stem cell therapy in a neurodegenerative brain. There are molecular factors which play key roles in controlling the differentiation; maturation and survival of stem cells which must be more thoroughly 
understood before the clinical application of stem cells are safely administered in such patients.

Therefore, in order to achieve a successful future outcome both in the clinic setting and in the patient's post-rehabilitant life; it is hoped that several of the discussed innovating strategies should be expanded on in terms of generating more data from animal models. Long-term and large scale multicentre clinical studies are also required to determine further, the precise therapeutic effects of cellular and non-cellular based treatment of neurological traumas and diseases. Not only will this allow methods to be more promising, but the outcome will also play a role in applying a multi-factorial approach that compliments synthetically produced nerve scaffolds that are capable of remodelling nerves, creating a supportive environment for neurogenesis alone or in the presence of combined cellular, surgical and therapeutic techniques.

\section{Concluding Remarks}

As this paper addresses a critical presentation on novel breakthroughs in the current status of adult neurogenesis; it can be seen that a greater understanding of molecular mechanisms, neuronal development, and cell plasticity are still urgently required to benchmark against several available high-potential therapies. Furthermore, novel insights behind connecting newly generated circuits can help revolutionise strategies for effective treatment of neurological pathologies. Therefore, the ultimate treatment goal should not only be to utilize therapy for the management of brain pathologies, but to also successfully apply the concept of neurogenesis and neural grafting throughout the brain, CNS and PNS; to achieve maximal recovery of the patient, and to help them regain their full mechanical and nervous system function.

\section{Abbreviations}

$\mathrm{A} \beta$, Amyloid- $\beta$ protein; $\alpha$-syn, alpha-synuclein $\mathrm{AD}$, Alzheimer's Disease; BBB, Blood Brain Barrier; BrdU, Bromodeoxyuridine; CNS, Central Nervous System; CAG, Cytosine-Adenine-Guanine; CSF, Cerebrospinal Fluid, CVO, Circumventricular Organ; DA, Dopamine; ePTFE, Expanded Polytetrafluoroethylene; GABA, gamma-Aminobutyric acid; GF, Growth Factors; HA, Hyaluronic Acid; HD, Huntington's Disease; iPSCs, induced Pluripotent Stem Cells; LB, Lewy Bodies; MS, Multiple Sclerosis; MSC, Mesenchymal Stem Cell; NGC, Nerve Guided Conduit; NSPC, Neural stem/progenitor cells; NSC, Neural Stem Cells; NT, Neurofibrillary Tangles; OB, Olfactory Bulb; PBS, Phosphate Buffered Saline; PD, Parkinson's Disease; PN, Parkinson's Neuritis; PNI, Peripheral Nerve Injury; PRP, Platelet Rich Plasma; RMS, Rostral Migratory Stream; SC, Schwann Cell; SCM, Scanning Electron Microscopy; SEL, Subendymal layer; SEM, Scanning Electron Microscope; SGZ, Subgranular Zone; SN, Substantia Nigra; SSRI, Selective Serotonin Reuptake Inhibitors; SVZ, Subventricular Zone; TBI, Traumatic Brain Injury

\section{References}

[1] Aurand, E. R., Lampe, K. J., Bjugstad, K. B. (2012), Defining and designing polymers and hydrogels for neural tissue engineering, Neuroscience Research; 72, 199-213

[2] Bhasin, A., Srivastava, M. V. P., Mohanty, S., Bhatia, R., Kumaran, S. S. (2013), Stem cell therapy: A clinical trial of stroke, Clinical Neurology and Neurosurgery; 115, 1003-1008

[3] Blaya, M. O., Tsoulfas., Bramlett, H. M., Dietrich, W. D. (2015), Neural Progenitor Cell Transplantation Promotes Neuroprotection, Enhances Hippocampal Neurogenesis, and Improves Cognitive Outcomes after Traumatic Brain Injury, Experimental Neurology; 264, 67-81

[4] Braun, S. M. G., Pilz, G. A., Machado, R. A. C., Moss, J., Becher, B., Toni, N., Jessberger, S. (2015), Programming Hippocampal Neural Stem/Progenitor Cells into Oligodendrocytes Enhances Remyelination in the Adult Brain after Injury, Cell Reports; 11, 1679-1685

[5] Christie, K. J., Tumley, A. M. (2013), Regulation of endogenous neural stem/progenitor cells for neural repairfactors that promote neurogenesis and gliogenesis in the normal and damaged brain, Frontiers in Cellular Neuroscience; 6, 1-18

[6] Cunha, C., Panseri, S., Antonini, S. (2011), Emerging nanotechnology approaches in tissue engineering for peripheral nerve regeneration, Nanomedicine: Nanotechnology, Biology, and Medicine; 7, 50-59

[7] Deumens, R., Bozkurt, A., Meek, M. F., Marcus, M. A. E., Joosten, E. A. J., Weis, J., Brook, G. A., (2010), Repairing injured peripheral nerves: Bridging the gap, Progress in Neurobiology; 92, 245-276

[8] Diez-Tejedor, E., Gutierrez-Fernandez, M., Martinez-Sanchez, P., Rodriguez-Frutos, B., Ruiz-Arez, G., Lara, M. L., Gimeno, B. F. (2014), Reparative Therapy for Acute Ischemic Stroke with Allogeneic Mesenchymal Stem Cells from Adipose Tissue: A Safety Assessment A Phase II Randomized, Doubleblind, Placebo-controlled, Single-center, Pilot Clinical Trial, Journal of Stroke and Cerebrovascular Diseases, 23, 26942700

[9] Drago, D., Cossetti, C., Iraci, N., Gaude, E., Musco, G., Bachi, A., Pluchino, S. (2013), The stem cell secretome and its role in brain repair, Biochimie; 95, 2271-2285

[10] Emsley, J. G., Mitchell, B. D., Kempermann, G., Macklis, J. D. (2005), Adult neurogenesis and repair of the adult CNS with neuralprogenitors, precursors, and stem cells, Progress in Neurobiology; 75, 321-341

[11] Faigle, R., Song, H. (2013), Signalling mechanisms regulating adult neural stem cells and neurogenesis, Biochim Biophys Acta $; 1830,2435-2448$

[12] Fairbairn, N. G., Meppelink, A. M., Ng-Glazier, J., Randolph, M. A., Winograd, J. M. (2015), Augmenting peripheral nerve regeneration using stem cells: A review of current opinion, World Journal Stem Cells; 26, 11-26

[13] Gu, X., Ding, F., Yang, Y., Liu, J (2011), Construction of tissue engineered nerve grafts and their application in peripheral nerve regeneration, Progress in Neurobiology; 93, 204-230 
[14] Hou, S., Xu., Q., Tian, W., Cui, F., Cai, Q., Ma, J., Lee, I. S. (2005) The repair of brain lesion by implantation of hyaluronic acid hydrogels modified with laminin, Journal of Neuroscience Methods; 148, 60-70

[15] Jiang, X., Lim, S. H., Mao, H. Q., Chew, S. Y. (2010), Current application and future perspectives of artificial nerve conduits, Experimental Neurology; 223, 86-101

[16] Kuffler, D. P., (2014), An assessment of current techniques for inducing axon regeneration and neurological recovery following peripheral nerve trauma, Progress in Neurobiology; $116,1-12$

[17] Lepousez, G., Nissant, A., Lledo1, P., (2015), Adult Neurogenesis and the Future of the Rejuvenating Brain Circuits, Neuron; 86, 387-401

[18] Li, X., Katsanevakis, E., Liu, X., Zhang, N., Wen. (2012), Engineering neural stem cell fates with hydrogel design for central nervous system regeneration, Progress in Polymer Science; 37, 1105-1129

[19] Lin, R., Cai, J., Nathan, C., Wei, X., Schleidt., Rosenwasser, R., Iacovitti, L. (2015), Neurogenesis is enhanced by stroke in multiple new stem cell niches along the ventricular system at sites of high BBB permeability, Neurobiology of Disease; 74, 229-239

[20] Lin, R., Iacovitti, L. (2015), Classic and novel stem cell niches in brain homeostasis and repair, Brain Research; 1628, $327-$ 342

[21] Loubinoux, I., Demain, B., Davoust, C., Plas, B., Vaysse, L. (2014), Stem cells and motor recovery after stroke, Annals of Physical and Rehabilitation Medicine; 57, 499-508

[22] Ming, G., and Song, H. (2012), Adult Neurogenesis in the Mammalian Brain: Significant Answers and Significant Questions, Neuron; 70, 687-702

[23] Musson, D. S., McIntosh, J., Callon, K. E., Chhana, A., Dunbar, P. R., Naot, D., Cornish, J. (2013), The need for thorough in vitro testing of biomaterial scaffolding: two case studies, Procedia Engineering; 59, 138-143

[24] Pabari, A., Yang, S. Y., Mosahebi, A., Seifalian, A. M. (2011), Recent advances in artificial nerve conduit design: Strategies for delivery of luminal fillers, Journal of Controlled Release; $156,2-10$
[25] Regnell, C. E., Hildrestrand, G. A., Sejersted, Y., Medin, T., Moldestad, O., Rolseth, V., Krokeide, S. Z., Suganthan, R., Luna, L., Bjøra, M., Bergersen, L. H., (2010), Hippocampal Adult Neurogenesis Is Maintained by Neil3-Dependent Repair of Oxidative DNA Lesions in Neural Progenitor Cells, Cell Reports; 2, 503-510

[26] Reyes, O., Sosa, I. J., Santiago, J., Kuffler, D. P., (2007), A novel technique leading to complete sensory and motor recovery across a long peripheral nerve gap, Puerto Rico Health Sciences Journal; 26, 225-228

[27] Ruan, R., Lau, B. W., Wang, J., Huang, L., Zhu Ge, Q., Wang, B., Jin, K., So, K., (2014), Neurogenesis in neurological and psychiatric diseases and brain injury: From bench to bedside, Progress in Neurobiology; 115, 116-137

[28] Subramanian, A., Krishnan U. M., Sethuraman, S. (2009), Development of biomaterial scaffold for nerve tissue engineering: Biomaterial mediated neural regeneration, Journal of Biomedical Science; 16, 1-11

[29] Vishwakarma, S. K., Bardia, A., Tiwari, S. K., Paspala, S. A. B., Khan, A. A., (2014), Current concept in neural regeneration research: NSCs isolation, characterization and transplantation in various neurodegenerative diseases and stroke: A review, Journal of Advanced Research; 5, 227-294

[30] Xiong, Y., Mahmood, A., Chopp, M. (2010), Angiogenesis, neurogenesis and brain recovery of function following injury, Current Opinion in Investigational Drugs; 11, 298-308

[31] Yao, S., Liu, X., Wang, X., Merolli, A., Chen, X., Cui, F. (2013), Directing neural stem cell fate with biomaterial parameters for injured brain regeneration, Progress in Natural Science: materials International; 23, 103-112

[32] Yoshikawa, G., Momiyama, T., Oya, S., Takai, K., Tanaka, J., Higashiyama, S., Saito, N., Kirino, T., Kawahara, N., Induction of striatal neurogenesis and generation of regionspecific functional mature neurons after ischemia by growth factors, Journal of Neurosurgery; 113, 835-850

[33] Zhao, C., Deng, W., Gage, F. H. (2008), Mechanism and Functional Implications of Adult Neurogenesis, Cell; 132, $645-660$ 